\title{
Detection of the whistlers in the VLF-spectrograms
}

\author{
Ekaterina Malysh ${ }^{1, \star}$ and Nina Cherneva ${ }^{2}$ \\ ${ }^{1}$ Vitus Bering Kamchatka State University, \\ 683031, Petropavlovsk-Kamchatsky, Russia \\ ${ }^{2}$ Institute of Cosmophysical Researches and Radio Wave Propagation FEB RAS, \\ Paratunka, Kamchatsky Kray, Russia
}

\begin{abstract}
The whistlers pattern recognition algorithm in the spectrogram of VLFradiation and algorithm of the filtering of pulse signals in the spectrograms are proposed.
\end{abstract}

\section{Introduction}

The whistlers it is a large part of the natural electromagnetic emission. They are formed as a result of frequency dispersion of the lightning pulse in the propagation of this pulse in the magnetospheric plasma. Whistlers are manifested in the spectrograms of VLF-signals as a structures (patterns) which having a characteristic curving shape [1]. The form of the dispersion curve is determined by the plasma parameters, so whistlers are natural markers of status Earth's plasmasphere [2], and their monitoring is a promising area of research of space weather.

The monitoring of whistlers on a planetary scale is carried stations of AWDANet, which are coordinated within the framework of the international project "Plasmon" [3]. One of the receivers of the AWDANet operates in site "Karymshina" of the Institute of Cosmophysics Research and Radio Wave Propagation (IKIR) FEB RAS.

The monitoring of whistlers in the IKIR also is conducted by means of the complex of its own design, which implements the automatic whistlers recognition system [4]. However, this system does not allow the detection of whistlers in real-time mode, which requires the development of new recognition systems.

In this paper, we describe an algorithm for image recognition of the whistlers in the spectrograms. Initial data for the problem of recognition in monitoring system are 15-minute wav-file of the VLFsignal. Viewed algorithm processes a 4-seconds segment of the file. The main idea of the algorithm is based on recognition "rectified" images and was described earlier in [5].

\section{Median filtering of the spectrograms}

Let us consider this segment of the spectrogram as a two-dimensional array $\mathbf{A}=\left\{A_{i j}\right\}, i=0, \ldots, N-1$, $j=0, \ldots, M-1$, where $N=513$ and $M=172$. The index $i$ enumerates discrete frequencies $f_{i}$, and index $j$ enumerates discretes $t_{j}$. An example of such a spectrogram containing one of whistler, shown in Fig. 1 top.

\footnotetext{
^e-mail: roswell-47@mail.ru
} 

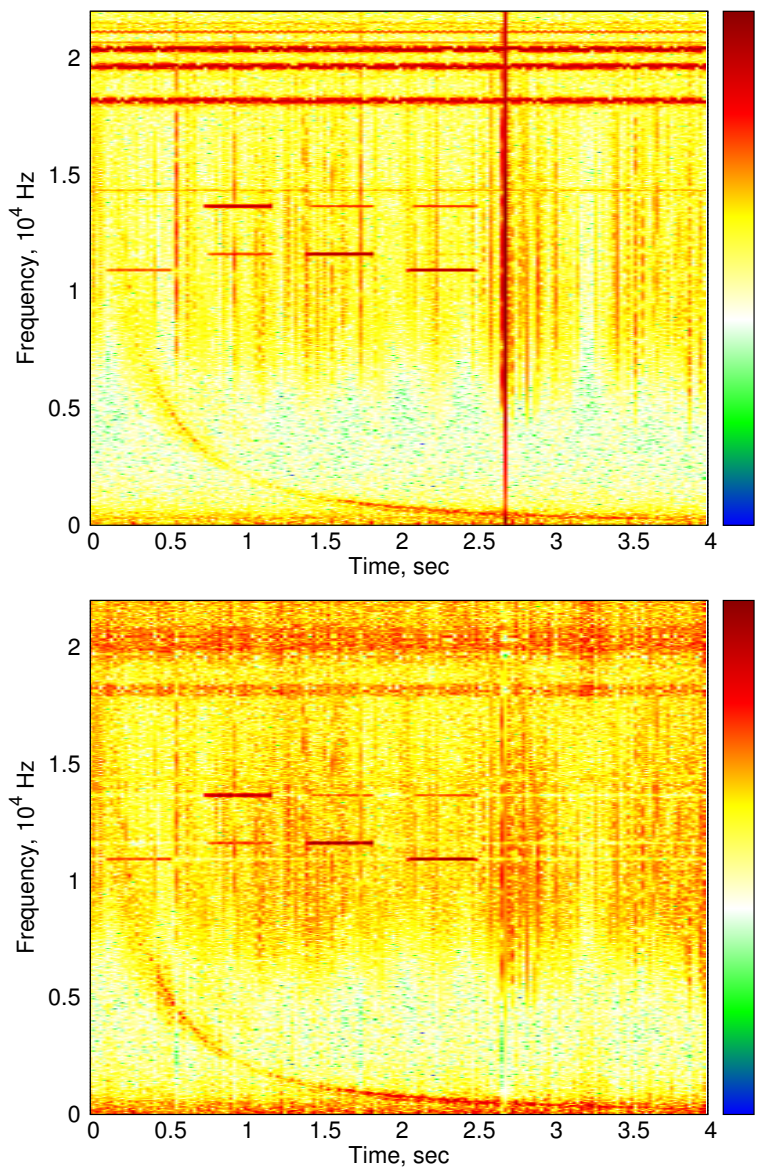

Figure 1. The spectrogram of VLF signal segment containing whistler: initial (top panel) and after median filtering (bottom panel).

The problem of whistler detection may be regarded as a problem of pattern recognition in a twodimensional image. The desired pattern is a curve with a characteristic form defined in the plane $(t, f)$ by the ratio $t-t_{0} \sim f^{-1 / 2}[1]$.

From Fig. 1 can clearly see that in addition to whistler in the analyzed signal there are $\delta$-like components (lightning pulses, so-called atmospherics) and narrow-band components. And those and others are noise for the problem of recognition. They are appear in the spectrogram in the form of lengthy linear structures, vertical and horizontal, respectively.

There is a need, above all, to quell this noise structures, and select a statistically significant points of the histogram.

It is well known, that the effective tool for cancellation lengthy noise in the images is the median filtering [6]. The median filters belongs to a class of nonlinear filters and keeps the sharpness of the image, as they are based on the order statistics, which are robust to strong emissions in data. The paper [7] was offered a modified median filtering algorithm, which allows to suppress lengthy vertical 
noise structures in satellite images. This algorithm has been adapted by us to solve the problem of cancellation lengthy vertical and horizontal structures to the spectrograms.

First, we make two one-dimensional arrays $\mathbf{X}$ and $\mathbf{Y}$, whose elements are the average values of the original spectrogram array in rows and columns, respectively:

$$
X_{i}=\frac{1}{N} \sum_{k=0}^{M-1} A_{i k}, \quad Y_{j}=\frac{1}{M} \sum_{k=0}^{N-1} A_{k j} .
$$

As a result, the each vertical noise structure is concentrated in several neighboring elements of array $\mathbf{X}$, and the each horizontal - in several neighboring elements array $\mathbf{Y}$. The strong emissions in these arrays will correspond to filterable noise structures.

Then, each of these arrays is subjected to one-dimensional median filtering:

$$
\begin{aligned}
& \hat{X}_{i}=\operatorname{median}\left\{X_{i+k} \mid \quad k=-L_{X}, \ldots, L_{X}\right\}, \quad i=L_{X}, \ldots, N-L_{X}-1, \\
& \hat{X}_{i}=\hat{X}_{L_{X}}, \quad i=0, \ldots, L_{X}-1, \\
& \hat{X}_{i}=\hat{X}_{N-L_{X}-1}, \quad i=N-L_{X}, \ldots, N-1, \\
& \hat{Y}_{j}=\operatorname{median}\left\{Y_{j+k} \mid \quad k=-L_{Y}, \ldots, L_{Y}\right\}, \quad j=L_{Y}, \ldots, M-L_{Y}-1, \\
& \hat{Y}_{j}=\hat{Y}_{L_{Y}}, \quad j=0, \ldots, L_{Y}-1, \\
& \hat{Y}_{j}=\hat{Y}_{M-L_{Y}-1}, \quad j=M-L_{Y}, \ldots, M-1,
\end{aligned}
$$

where $2 L_{X}+1$ and $2 L_{Y}+1$ are the lengths of filter masks. Then arrays noise is calculated $\Delta \mathbf{X}=\mathbf{X}-\hat{\mathbf{X}}$ and $\Delta \mathbf{Y}=\mathbf{Y}-\hat{\mathbf{Y}}$.

Filter spectrogram $\hat{\mathbf{A}}$ define as $\hat{A}_{i j}=A_{i j}-\left(\Delta X_{i}+\Delta Y_{j}\right) / 2$. This spectrogram is shown in Fig. 1 bottom. It can be seen that the proposed algorithm of median filtering images really effectively suppresses atmospherics and narrowband noise, and the pattern of whistler remains clear. The short radio signals $(\sim 0.5 \mathrm{sec}$.) also remained.

\section{Selection of significant points of the spectrogram}

On next step, we selected of significant points for each column in the filtered spectrogram. We consider the squares of elements of the column as the Schuster periodogram corresponding segment of the VLF signal, and checks the hypothesis that this segment is white noise on the significance level $\alpha$ [8].

To do this, we calculate for each $j$-th column of the spectrogram $\hat{\mathbf{A}}$ variance $\sigma_{j}^{2}$ through its spectral representation:

$$
\sigma_{j}^{2}=2 \sum_{i=0}^{N-1} \hat{A}_{i j}^{2}
$$

Furthermore, if $\hat{A}_{i j}^{2}<-\sigma_{j}^{2}(\ln \alpha) / N$, then the point $\hat{A}_{i j}$ is considered noise, otherwise case - significant.

Of the pairs of indices $(i, j)$ of significant elements we formed the dynamic one-dimensional array $\mathbf{P}$ with elements $P_{m}=\left(i_{m}, j_{m}\right)$, whose length is equal to the number of significant elements of the spectrogram. 
This is done to acceleration of processing. The thing is further recognition algorithm is working with arrays of $\mathbf{P}=\left\{P_{m}\right\}, \mathbf{t}=\left\{t_{j}\right\}$ and $\mathbf{f}=\left\{f_{i}\right\}$, but not with points of the spectrogram. The element $P_{m}$ corresponds to the point $\hat{A}_{i_{m} j_{m}}$ on the filtered spectrogram. Next we are identifying $P_{m}$ and $\hat{A}_{i_{m} j_{m}}$.

The transition to these arrays provides a significant reduction in the volume of data being processed further. Analysis of typical spectrograms showed that the length of the array $\mathbf{P}$ is approximately by an order less than that of the initial spectrogram.

On top panel of the Fig. 2 by points shows the elements selected for the spectrogram $\hat{\mathbf{A}}$. It can be seen that the image of whistler is well looked through after selection.
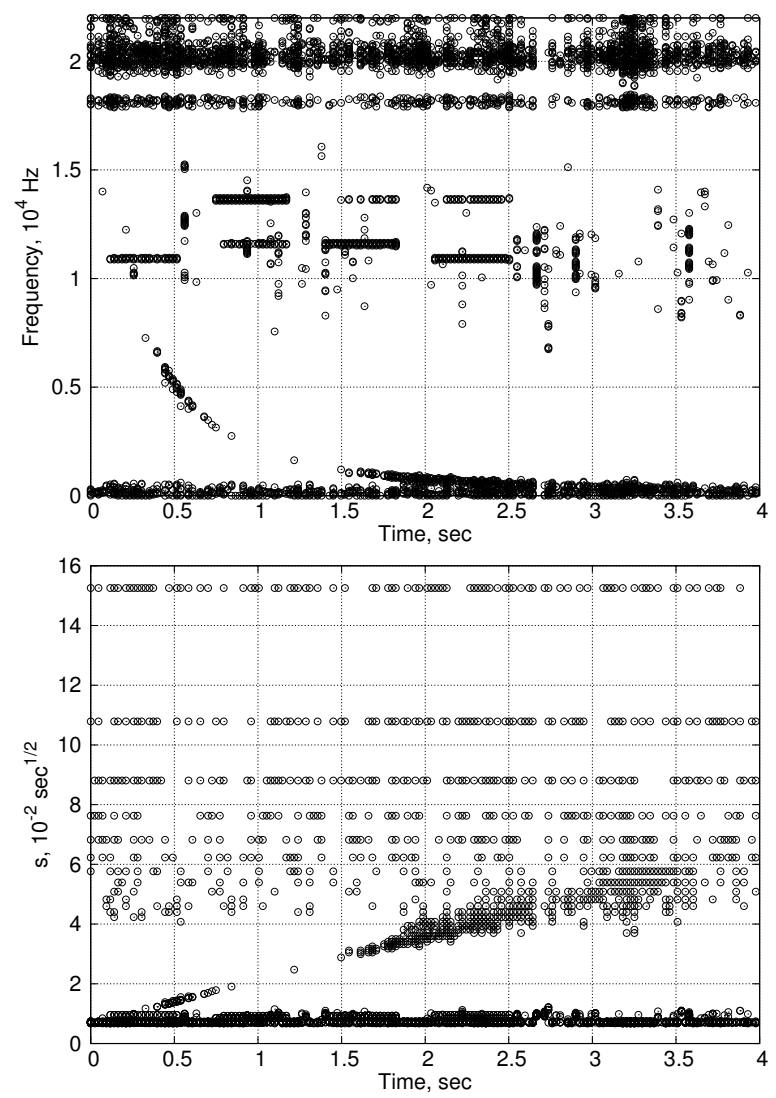

Figure 2. Significant points of the spectrogram in the plane $(t, f)$ (top panel) and in the plane $(t, s)$ (bottom panel).

\section{Recognition algorithm}

From the theory of VLF waves propagation in the magnetospheric plasma is known [1], that the pattern of whistler, has come at the time $\tau$, is well described by the equation

$$
t-\tau=D / \sqrt{f}
$$


where the coefficient $D>0$ is called dispersion. From (4) it is clear that, if we introduce the transformation $s=f^{-1 / 2}$, then in the plane $(t, s)$ pattern of the whistler is rectified, and Eqs. (4) corresponds to the equation in this plane $t-D s-\tau=0$, i.e. equation inclined straight, which forming a non-zero acute angle with the positive direction of the time axis. The points $Q_{m}=\left(t_{j_{m}}, s_{i_{m}}\right)$ corresponding to the elements of the $P_{m}=\left(i_{m}, j_{m}\right)$ are shown in bottom panel of Fig. 2 .

The straight line recognition problem is algorithmically simpler, than the recognition of the curve. Therefore, more convenient to detected whistler in the plane $(t, s)$. Then, an indication of the presence of the whistler (whistlers) is a grouping of points near the straight line (lines) in this plane. And we are talking only about the slanted lines, as the remains of the narrow-band noise and radio signals after median filtering and selection of significant elements continue to manifest themselves in the form of horizontal rectilinear structures.

Of the many variants of the equations of this direct lines, is best suited for our purposes

$$
t \sin \varphi-s \cos \varphi-d=0
$$

where $\varphi$ - angle formed by a straight line with the axis of time, and $d$-generalized distance from the line to the origin. The distance it takes a positive, if the line crosses the $s$-axis in a positive point, and negative otherwise.

Processing a large number of spectrograms showed that the real value $\varphi \in\left[1^{0} ; 10^{0}\right]$. It is also clear that $d \geq d_{\min }=-\max \left\{t_{j_{m}}\right\}$ and $d \leq d_{\max }=\max \left\{s_{i_{m}}\right\}$.

A pencil of lines passing through the point $Q_{M}$, defined by the equation

$$
t_{j_{m}} \sin \varphi-s_{i_{m}} \cos \varphi=d,
$$

describing the line in the area $1^{0}<\varphi<10^{0}, d_{\min } \leq d \leq d_{\max }$ in the plane $(\varphi, d)$. Then the straight line (whistler pattern), passing through several points of $Q_{m}$, will correspond to the point of intersection of these lines in $(\varphi, d)$. Therefore, an indication of the proximity of the points to a one straight line in the plane $(t, s)$ is the existence of a small area in the plane $(\varphi, d)$, that is crossed these a line. Moreover, smaller the area, the closer to straight line arranged the point in the $(t, s)$, and inversely.

We introduce a analytical grid $\left\{\varphi_{k}\right\}$ and $\left\{d_{l}\right\}$ on the set of possible values of angles and distances. For each point $Q_{m}$ and every angle $\varphi_{k}$ from the equation (6) define the distance $d$ and rounded up to the nearest $d_{l}$.

The result is a two-dimensional histogram of the pairs $(\varphi, d)$ distribution for the points $Q_{m}$. If the value histogram for the pair $\left(\varphi_{k}, d_{l}\right)$ is large, then a lot of $Q_{m}$ is near the corresponding line. Thus, each significant maximum of the histogram can be associated with whistler.

Selection of significant maximums may also be performed as described above on the basis of the Schuster theorem.

\section{Conclusion}

In this paper we describe an algorithm for whistlers pattern recognition in the spectrogram of VLF radiation, consisting of four stages: median filtering; selection of significant points; coordinate transformation with a view to "rectifying" the whistler pattern; recognition "rectified" whistler.

The recognition algorithm testing showed that processing of 225 spectrograms for 15-minute wavfile requires less than 4 minutes, which ensures significant reserve of processing time. Now we develop a neural network to detect whistlers in automatic mode on the basis of the described algorithm. 


\section{References}

[1] B. N. Gershman, V. A. Ugarov, Sov. Phys. Usp., 3, A07222 (1961)

[2] J. Lichtenberger, Jour. Geophys. Res.: Space Physics, 114, A07222 (2009)

[3] http://plasmon.elte.hu/

[4] N. V. Cherneva, V. P. Sivokon', I. V. Agranat, Problems of Geocosmos. Proc. of the 9th Int. Conf., 102-106 (2012)

[5] E. A. Malysh, Bulletin KRASEC. Phys.\&Math. Sci., 11:2, 82-87 (2015)

[6] R. C. Gonzales, R. E. Woods, Digital Image Processing (Prentice-Hall Inc., Upper Saddle River, 2002), 782.

[7] Yu. M. Gektin, A. A. Zaytsev, Trudy MFTI, 6:4, 103-106 (2014)

[8] S. L. Marpl-jr., Digital Spectral Analysis: With Applications (Prentice-Hall Inc., New Jersey, 1987), 523 